\title{
Expression of glutamine synthetase in Tegillarca granosa (Bivalvia, Arcidae) hemocytes stimulated by Vibrio parahaemolyticus and lipopolysaccharides
}

\author{
Y.B. Bao' ${ }^{1}$ L. Li ${ }^{2}$, M.X. Ye', Y.H. Dong ${ }^{1}$, W.X. Jin ${ }^{1}$ and Z.H. Lin ${ }^{1}$ \\ ${ }^{1}$ College of Biological and Environmental Sciences, \\ Zhejiang Wanli University, Ningbo, Zhejiang, China \\ ${ }^{2}$ Institute of Oceanology, Chinese Academy of Sciences, \\ Qingdao, Shandong, China \\ Corresponding authors: Z.H. Lin / Y.B. Bao \\ E-mail: zhihua9988@126.com / bobbao2001@gmail.com
}

Genet. Mol. Res. 12 (2): 1143-1154 (2013)

Received July 27, 2012

Accepted October 9, 2012

Published April 10, 2013

DOI http://dx.doi.org/10.4238/2013.April.10.9

\begin{abstract}
The blood cockle, Tegillarca granosa, is a widely consumed clam in the Indo-Pacific region. Glutamine synthetase (GS) is an enzyme that plays an essential role in the metabolism of nitrogen by catalyzing the condensation of glutamate and ammonia to form glutamine. We identified the GS of T. granosa (Tg-GS) from hemocytes by 3'- and 5'-rapid amplification of cDNA ends (RACE)-PCR. The fulllength cDNA consisted of $1762 \mathrm{bp}$, with a 1104-bp open reading frame encoding 367 amino acids. Sequence comparison showed that Tg-GS has homology to GS of other organisms, with $79.78 \%$ identity with GS from the Pacific oyster Crassostrea gigas, $71.98 \%$ identity with GS from the zebrafish Danio rerio, and $68.96 \%$ identity with human Homo sapiens GS. A C-beta-Grasp domain and an N-catalytic domain were identified in Tg-GS, indicating that Tg-GS should be classified as a new member of the GS family. A quantitative RT-PCR assay was used to detect mRNA expression of Tg-GS in five different tissues. Higher levels
\end{abstract}


of mRNA expression of GS were detected in the tissues of hemocytes and the mantle. Up-regulation of GS by challenge with the bacteria Vibrio parahaemolyticus and with bacterial wall lipopolysaccharides showed that GS plays a role in anti-bacterial immunity. We conclude that pathogen infection significantly induces expression level of TgGS, and that activation of GS influences the immune response of $T$. granosa by increasing glutamine concentration.

Key words: Vibrio parahaemolyticus; Messenger RNA expression; Immune response; Tegillarca granosa; Glutamine synthetase

\section{INTRODUCTION}

Glutamine synthetase (GS) (EC 6.3.1.2) is an enzyme that plays an essential role in the metabolism of nitrogen by catalyzing the condensation of glutamate and ammonia to form glutamine: glutamate $+\mathrm{ATP}+\mathrm{NH}_{3} \rightarrow$ glutamine $+\mathrm{ADP}+$ phosphate $+\mathrm{H}_{2} \mathrm{O}$ (Eisenberg et al., 1987). GS is found in a wide range of organisms, including microorganisms, plants, and animals. It is a ubiquitous enzyme involved in complex cellular functions, including nitrogen metabolism, recycling of the neurotransmitter glutamate, synthesis of glutamine for the production of amino acids and sugars, and glucosamine-6-phosphate regeneration (Myohara et al., 2006). Three classes of GS are found in diverse organisms (Kumada et al., 1993). Class I enzymes are oligomers of 12 identical subunits specific to prokaryotes. Class II enzymes have 8 identical subunits and are found in eukaryotes and in bacteria belonging to the Rhizobiaceae, Frankiaceae, and Streptomycetaceae families (Shatters and Kahn, 1989). Class III enzymes have been found to date only in Bacteroides fragilis and Butyrivibrio fibrisolvens. They are hexamers of identical chains. Class III GS is much larger (about 700 amino acids) than class I GS (450 to 470 amino acids) or class II GS (350 to 420 amino acids) (Brown et al., 1994).

GS has been cloned from several vertebrates, including Chinese hamster (Cricetulus griseus; Hayward et al., 1986), rat (Rattus rattus; Fahrner et al., 1993), and rainbow trout (Oncorhynchus mykiss; Murray et al., 2003), and from invertebrates such as Pacific oyster (Crassostrea gigas; Tanguy et al., 2005), mosquito (Aedes aegypti; Smartt et al., 2001), and spiny lobster (Panulirus interruptus; Trapido-Rosenthal et al., 1993). Several species contain various isoforms of GS, which are encoded by multiple genes. These isoforms are expressed in diverse tissues or they play specific roles. Mitochondrial and cytosolic isozymes of Drosophila melanogaster GS have been identified (Caizzi et al., 1990). In fish, GS is a multifunctional enzyme, just as the product glutamine has many metabolic roles (Murray et al., 2003).

However, many species such as mammals have a single-GS gene encoding for one isoform (Kuo and Darnell Jr., 1989). In mollusca, only the GS from Pacific oyster has been isolated. However, few studies of the immune responses of GS in molluscs infected with bacterial antigens have been published. The aims of the present study were 1) to isolate and characterize the blood clam (Tegillarca granosa) GS (Tg-GS) complementary DNA (cDNA) and compare its deduced amino acid sequence with other known GSs, 2) to examine the expression of Tg-GS in various tissues, and 3) to evaluate Tg-GS expression in blood clams injected with Vibrio parahaemolyticus and lipopolysaccharide (LPS). 


\section{MATERIAL AND METHODS}

\section{cDNA library construction and expressed sequence tag (EST) analysis}

A cDNA library was constructed from the hemocytes of a bay scallop (Argopecten irradians) using a Creator Smart cDNA Library Construction Kit (Clontech, USA) and a TRIMMER-DIRECT kit (Evrogen, Russia). Random sequencing of the library using T7 primer yielded 2278 successful sequencing reactions. Basic Local Alignment Search Tool analysis of all the ESTs revealed that one EST of 554 bp was highly similar to GS in C. gigas (score, 219 bits; expect, 9E-56; identities, 75\%; GenBank No. CAD90162), and this EST was used to design primers for Tg-GS gene cloning.

\section{Cloning of the full-length cDNA of the Tg-GS gene}

Two gene-specific primers, Tg-GS-F1 and Tg-GS-R1, were designed based on the partial sequence data of GS (Table 1). The 3'-rapid amplification of cDNA ends (RACE)-PCR was performed with the gene-specific primer Tg-GS-F1 and universal vector primer T7. PCR was carried out with a program of 35 cycles of $94^{\circ} \mathrm{C}$ for $40 \mathrm{~s}, 58^{\circ} \mathrm{C}$ for $40 \mathrm{~s}$, and $72^{\circ} \mathrm{C}$ for $50 \mathrm{~s}$, and an extension of $72^{\circ} \mathrm{C}$ for $10 \mathrm{~min}$. The $5^{\prime}$-RACE-PCR was performed with the genespecific primer Tg-GS-R1 and universal vector primer T3. The PCR conditions were the same as those described above except for the annealing temperature $\left(58^{\circ} \mathrm{C}\right)$. The PCR products were cloned into the pMD18-T simple vector (TaKaRa, Japan) and sequenced in both directions with primers M13-47 and RV-M. The resulting sequences were verified and subjected to cluster analysis.

\begin{tabular}{|c|c|c|}
\hline Primers & Sequences $\left(5^{\prime}-3^{\prime}\right)$ & Application \\
\hline Tg-GS-F1 & ATCCTGGAACCACCACAAATAAA & 3'-RACE \\
\hline Tg-GS-R1 & GGTGCTGTGGGTTCAAAGTCTAA & 5'-RACE \\
\hline $\mathrm{T} 7$ & TAATACGACTCACTATAGG & 3'-RACE \\
\hline $\mathrm{T} 3$ & AATTAACCCTCACTAAAGGG & 5'-RACE \\
\hline M13-47 & CGCCAGGGTTTTCCCAGTCACGAC & Cloning sequence \\
\hline RV-M & GAGCGGATAACAATTTCACACAGG & Cloning sequence \\
\hline Tg-GS-F-real & GCCGTCTTGGTGGTAAATGC & qRT-PCR of GS \\
\hline Tg-GS-R-real & TACTGCCTCGTAGCGCCTCA & qRT-PCR of GS \\
\hline Tg-18srRNA-F-real & CTTTCAAATGTCTGCCCTATCAACT & qRT-PCR of $18 \mathrm{srRNA}$ \\
\hline Tg-18srRNA-R-real & TCCCGTATTGTTATTTTTCGTCACT & qRT-PCR of 8 srRNA \\
\hline
\end{tabular}

$\mathrm{RACE}=$ rapid amplification of cDNA ends; GS = glutamine synthase.

\section{Analysis of nucleotide and amino acid sequences}

The nucleotide and deduced amino acid sequence of Tg-GS cDNA were analyzed using DNAMAN 5.2.2. The GS sequences from various organisms were aligned through the National Center for Biotechnology Information Basic Local Alignment Search Tool program. A multiple sequence alignment was created with ClustalW [http://www.ebi.ac.uk/clustalw/ (accessed September 24, 2012)] and a phylogenetic tree of GS was drawn using MEGA 3.1 [http://www.megasoftware.net (accessed September 24, 2012)]. The signal peptide was pre- 
dicted using the SignalP program [http://www.cbs.dtu.dk/services/SignalP/ (accessed September 24, 2012)]. The calculated molecular mass and theoretical isoelectric point was predicated using a calculation of protein isoelectric point program [http://isoelectric.ovh.org/ (accessed September 24, 2012)].

\section{Animals and immune challenge}

Blood clams aged 2 to 3 years old were collected from a commercial farm (Ningbo, China) and kept in seawater at a temperature of $26^{\circ} \pm 1.0^{\circ} \mathrm{C}$ and a salinity of $30 \%$. To minimize individual variability, at least 60 clams were used in each experiment. In the V.parahaemolyticus challenge group, $20 \mu \mathrm{L}$ live $V$. parahaemolyticus suspended in phosphate-buffered saline (PBS; $2 \times 10^{8} \mathrm{cfu} / \mathrm{mL}, \mathrm{pH} 7.2$ ) was injected into the adductor muscle of each individual. Four individuals were randomly sampled $1.5,3,6,12,24$, and $48 \mathrm{~h}$ after injection. The clams in the LPS (L6511, Sigma, USA) challenge group were injected with $20 \mu \mathrm{L} 0.2 \mathrm{mg} / \mathrm{mL}$ drugs (diluted in $0.85 \% \mathrm{NaCl}$ ) per individual after the procedures described above. Unchallenged clams and 60 clams injected with $20 \mu \mathrm{L}$ PBS were used as the time 0 group (at $0 \mathrm{~h}$ ) and the control group, respectively.

\section{Tissue collection, RNA extraction, and cDNA synthesis}

Blood clams averaging $30 \mathrm{~mm}$ in shell length were collected from a clam farm in Ningbo, China, and acclimatized in seawater tanks $\left(10 \mathrm{~m}^{3}\right)$ for 1 week before processing. The seawater temperature was $18^{\circ} \pm 1.0^{\circ} \mathrm{C}$, and the salinity was $30 \%$ throughout the experiments. The seawater was changed every day. Hemocyte, hepatopancreas, gill, adductor muscle, gonad, and mantle tissues were collected from four scallops to investigate the tissue-specific expression of Tg-GS. Total RNA was extracted from tissues using the TRIzol reagent (Invitrogen) following the manufacturer protocol. The extracted RNA was then treated with RQ1 RNase-Free DNase (Promega) to remove contaminating DNA. cDNAs were synthesized from total RNA with M-MLV reverse transcriptase (Promega) and Oligo(dT).

\section{Quantification analysis of Tg-GS messenger RNA (mRNA) expression}

Quantitative reverse transcription (qRT)-PCR was performed on an Mx3000P system (Stratagene, USA) to characterize the tissue-specific expression of Tg-GS and temporal expression of Tg-GS in hemocytes of bay scallops injected with V. anguillarum and LPS. Two Tg-GS-specific primers, forward primer Tg-GS-F-real and reverse primer Tg-GS-R-real (see Table 1), were used to amplify a 194-bp fragment of Tg-GS. Primers Tg-18srRNA-Freal and Tg-18srRNA-R-real were used to amplify a 195-bp fragment of Tg-18srRNA as the internal control for qRT-PCR. All analyses were based on the the threshold cycle $\left(\mathrm{C}_{\mathrm{T}}\right)$ values of the PCR products, and a comparative $\mathrm{C}_{\mathrm{T}}$ method was used to analyze the expression level of Tg-18srRNA according to previous studies (Bao et al., 2009a,b). The data obtained from qRT-PCR analysis were subjected to an LSD $t$-test to determine the difference in mean values among treatments. The $\mathrm{P}$ value for significance was set at $\leq 0.05$. Statistical analysis was performed using the SPSS 16 software (IBM, USA). 


\section{RESULTS}

\section{cDNA library construction and EST analysis}

A normalized, full-length cDNA library was constructed from hemocytes of the blood clam. A total of 2278 ESTs from 5'-ends of the cDNA clones representing 1501 unigenes with an average length of $459 \mathrm{bp}$ were obtained from the library. Cluster analysis of these ESTs identified 1501 unique sequences containing 173 contigs and 1328 singletons. One EST was highly homologous to the GS gene from C. gigas (GenBank accession No. CAD90162).

\section{cDNA and deduced amino acid sequence of Tg-GS}

The 3'- and 5'-ends of Tg-GS were cloned using the SMART-RACE approach. The full-length Tg-GS cDNA sequence (GenBank accession No. HM778111) was 1762 bp, containing a 5'-untranslated region (UTR) of 69 nucleotides, a 1104-bp open reading frame encoding 367 amino acids, and a 3'-UTR of 593 nucleotides containing a stop codon (TAA) and a possible polyadenylation signal (AATAAA) 19 bp upstream of the polyadenylation tail (Figure 1). The calculated molecular mass of the deduced mature Tg-GS was $41.34 \mathrm{kDa}$, and the theoretical isoelectric point was 5.81. The amino acid sequences of Tg-GS were aligned with those of selected GS sequences from the National Center for Biotechnology Information database using the ClustalW method. The primary structure of Tg-GS showed similarity to the GS of four animals (Figure 2). The deduced amino acid sequence of Tg-GS showed identity with those of the C. gigas (79.78\%), honey bee (Apis mellifera; 65.30\%), zebrafish (Danio rerio; 71.98\%), and human (Homo sapiens; 68.96\%). Furthermore, Tg-GS contained 1) two principal domains, C-beta-Grasp domain and N-catalytic domain; 2) five conserved regions that are common to both prokaryotes and eukaryotes - ligands for $\mathrm{Mg}^{2+}$ ions; and 3) an ATP binding site (see Figure 2).

\section{Phylogenetic analysis of GSs}

Using the neighbor-joining method, a phylogenetic tree was constructed based on the amino acid sequences of selected animal GSs with two plant GSs as the outgroup (Figure 3; Table 2). All GSs clustered together as a subgroup and the plant GSs as the outgroup clustered into another subgroup. In the subgroup of GSs, Tg-GS was first clustered with GS from $C$. gigas and then formed a sister subgroup with worm and arthropod. Tg-GS was somewhat close to that of invertebrates such as worm (Enchytraeus japonensis), insect (A. mellifera), and shrimp (Fenneropenaeus chinensis) but was distant from that of fishes, amphibians, chicken, and mammals. Plant GSs as the outgroup from corn (Zea mays) and lettuce (Lactuca sativa) clustered together and then clustered with animal GSs at low bootstrap values.

\section{Quantitative analysis of Tg-GS gene expression}

Real-time quantitative PCR was used to quantify Tg-GS expression in hemocyte, adductor muscle, foot, digestive tract, gill, and mantle tissues. The amplification specificity for Tg-GS and Tg-18srRNA were determined by analyzing the dissociation curves. Only one 
peak appeared in the dissociation curves for both Tg-GS and Tg-18srRNA genes, indicating that the amplifications were specific. The mRNA transcript of Tg-GS could be detected in all examined tissues at different expression levels. The highest level of Tg-GS expression was detected in hemocytes, the next highest in mantle, and medium levels in adductor muscle, foot, and gill $(\mathrm{P}<0.01)$. The lowest level of Tg-GS expression was detected in the hepatopancreas $(\mathrm{P}<0.01$; Figure 4$)$. Expression profiles of Tg-GS in hemocytes after $V$. parahaemolyticus and LPS challenge are shown in Figure 5.

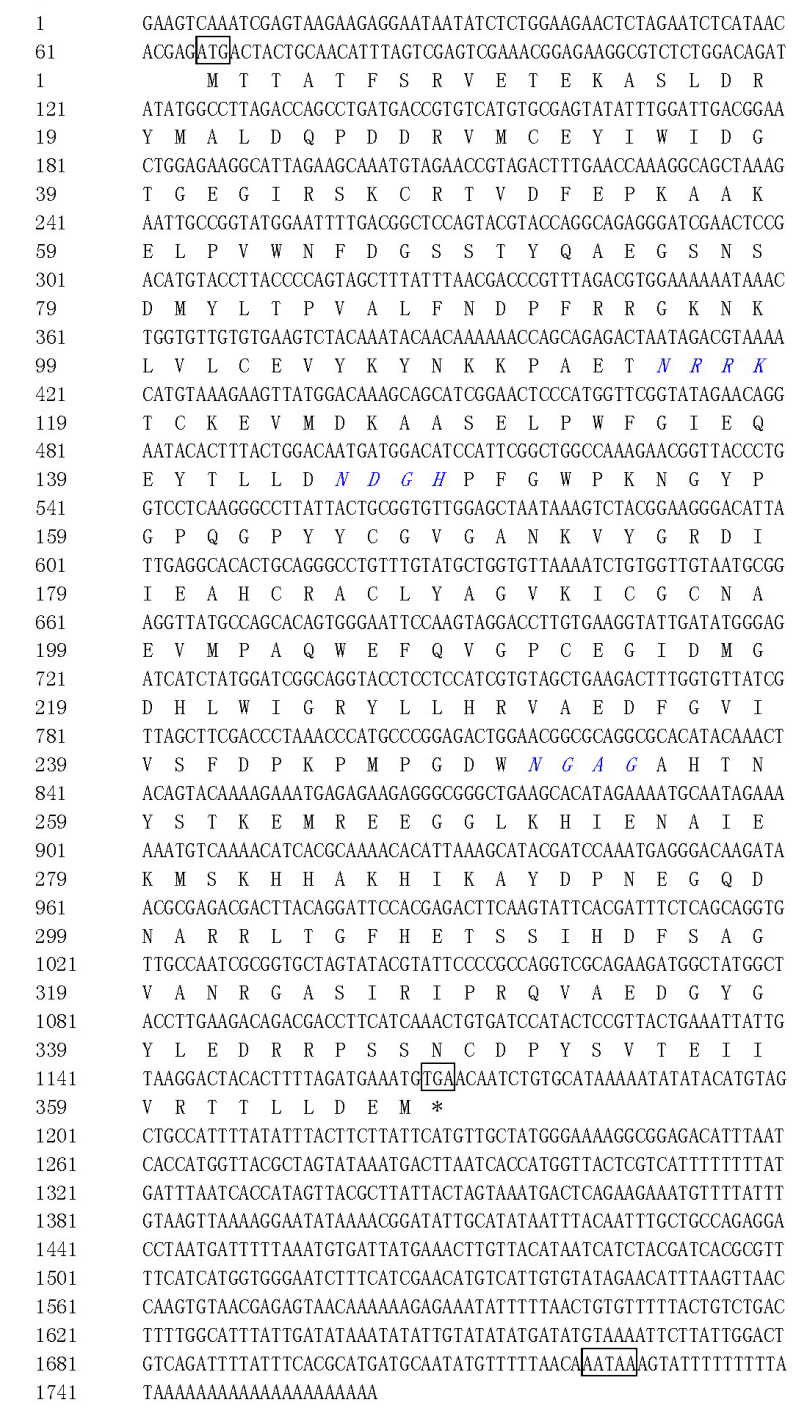

Figure 1. Nucleotide and deduced amino acid sequences of glutamine synthetase of Tegillarca granosa (Tg-GS). The letters in boxes are the start codon (ATG), the stop codon (TGA), and the polyadenylation signal sequence (ATTAAA). Two GS family signatures predicted by the InterPro Scan program are italicized. The stop codon is labeled with an asterisk. 


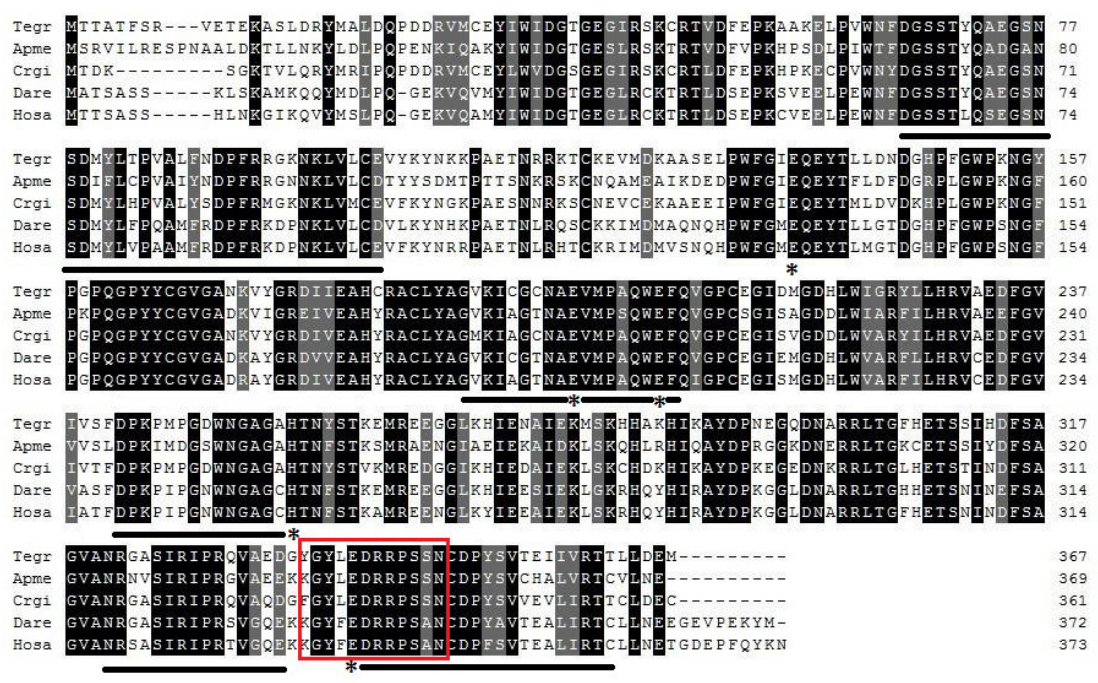

Figure 2. Multiple sequence alignments of glutamine synthetase of Tegillarca granosa (Tg-GS) with other four known GS amino acid sequences: T. granosa (Tegr, ADM94277), Apis mellifera (Apme, NP_001164445), Crassostrea gigas (Crgi, CAD90162), Danio rerio (Dare, NP 878286), Homo sapiens (Hosa, NP_001028228). Five conserved regions are underlined. Ligands for $\mathrm{Mg}^{2+}$ ions are indicated by asterisk. The ATP-binding sites are boxed.

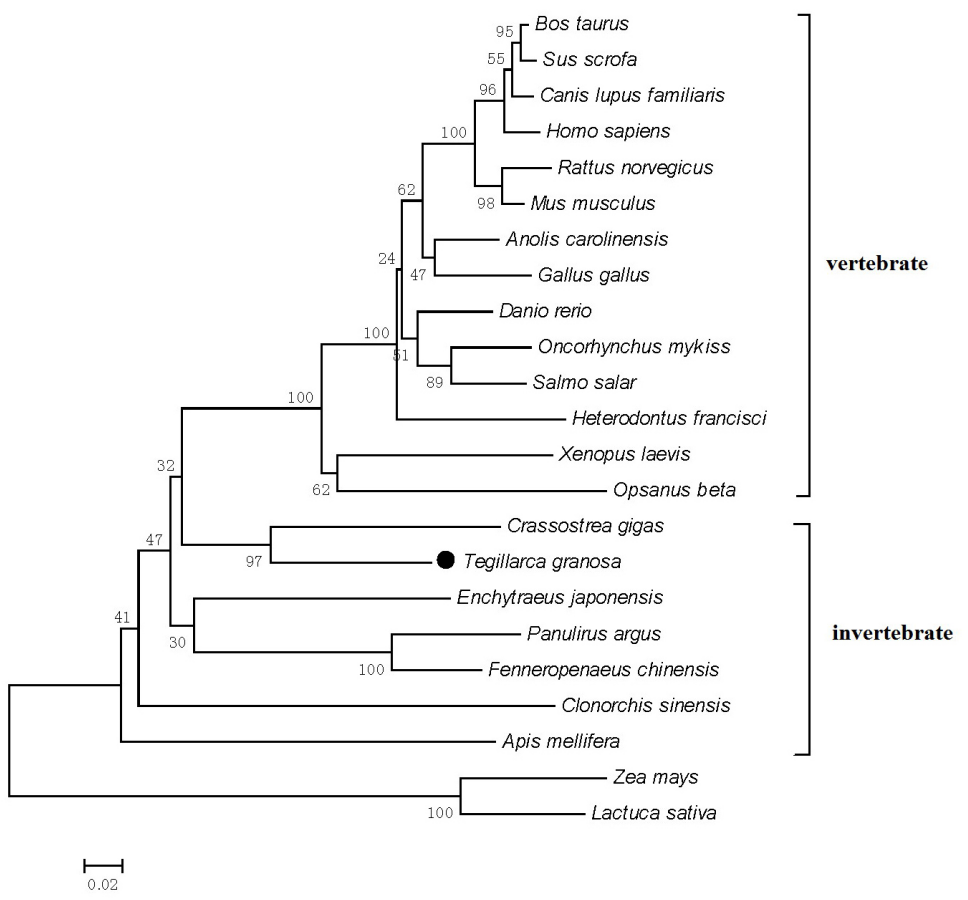

Figure 3. Phylogenetic tree of the glutamine synthase (GS) protein from 15 species reconstructed by the neighborjoining method with 1000 bootstrap trials by the MEGA 3.4 program. Numbers at each branch node represent the values given by bootstrap analysis. 
Table 2. Species and GenBank accession numbers of glutamine synthase sequences used for multiple alignment and phylogenetic analysis.

\begin{tabular}{|c|c|c|c|}
\hline Species & Common name & GenBank No. & Size (amino acids) \\
\hline Apis mellifera & Bee & NP_001164445 & 369 \\
\hline Anolis carolinensis & Anole & XP_003228795 & 381 \\
\hline Bos taurus & Bovine & NP_001035564 & 373 \\
\hline Canis lupus familiaris & Dog & NP_001002965 & 373 \\
\hline Clonorchis sinensis & Fluke & GAA 51709 & 364 \\
\hline Crassostrea gigas & Oyster & CAD92144 & 361 \\
\hline Danio rerio & Zebrafish & NP_878286 & 372 \\
\hline Enchytraeus japonensis & Worm & BAE93509 & 360 \\
\hline Fenneropenaeus chinensis & Shrimp & ACB59229 & 361 \\
\hline Gallus gallus & Chicken & NP_990824 & 373 \\
\hline Heterodontus francisci & Shark & CAD90162 & 348 \\
\hline Homo sapiens & Human & NP_001028228 & 330 \\
\hline Lactuca sativa & Lettuce & CAÄ42689 & 358 \\
\hline Mus musculus & Mouse & NP_032157 & 373 \\
\hline Oncorhynchus mykiss & Trout & NP_001117785 & 373 \\
\hline Opsanus beta & Toadfish & AAN777155 & 373 \\
\hline Panulirus argus & Lobster & AAA02583 & 361 \\
\hline Rattus norvegicus & Rat & NP_058769 & 373 \\
\hline Salmo salar & Salmon & NP_001134684 & 371 \\
\hline Sus scrofa & Pig & NP_999074 & 373 \\
\hline Tegillarca granosa & Clam & AAD 34721 & 367 \\
\hline Xenopus laevis & Frog & DAA00256 & 371 \\
\hline Zea mays & Corn & BAA03433 & 357 \\
\hline
\end{tabular}

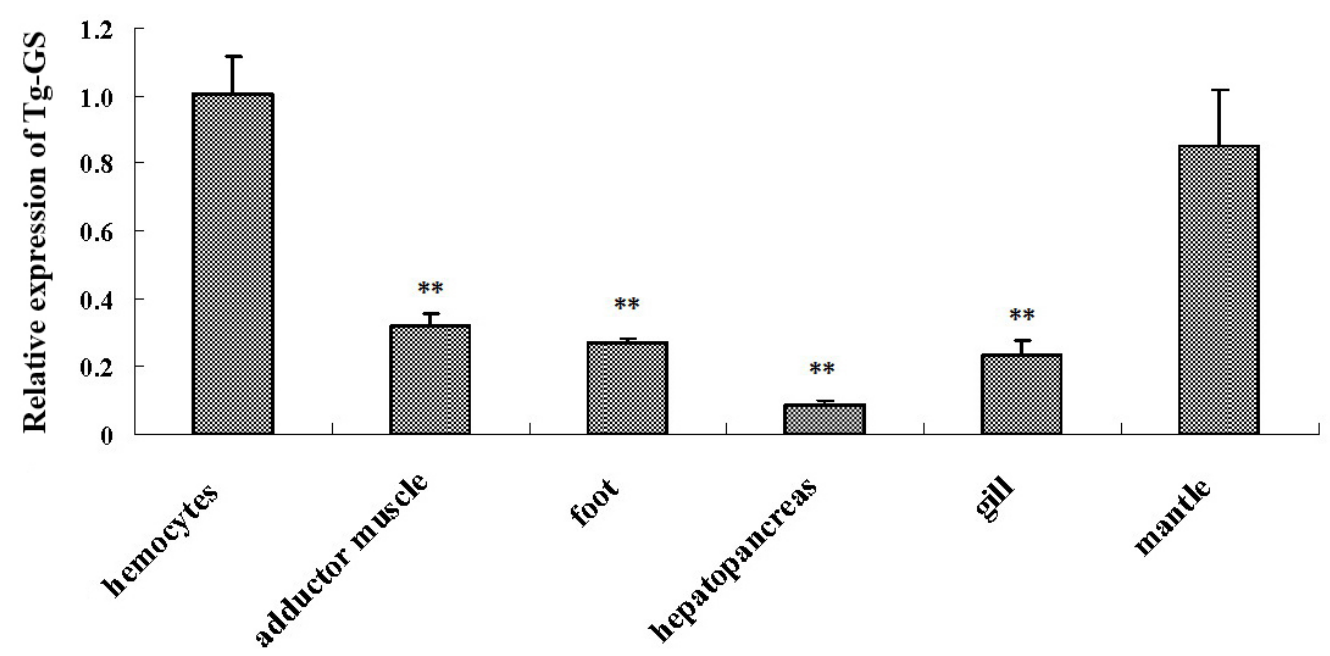

Figure 4. Real-time analysis of the amount of glutamine synthetase of Tegillarca granosa (Tg-GS) transcript relative to 18rRNA transcript in different tissues. The tissues, including hemocytes, adductor muscle, foot, hepatopancreas, gill, and mantle, were collected from bloody clams. Vertical bars represent means \pm standard deviation $(\mathrm{N}=3)$. Significant differences compared to the hemocytes are indicated with an asterisk at $\mathrm{P}<0.05$; very significant differences are indicated with two asterisks at $\mathrm{P}<0.01$. 


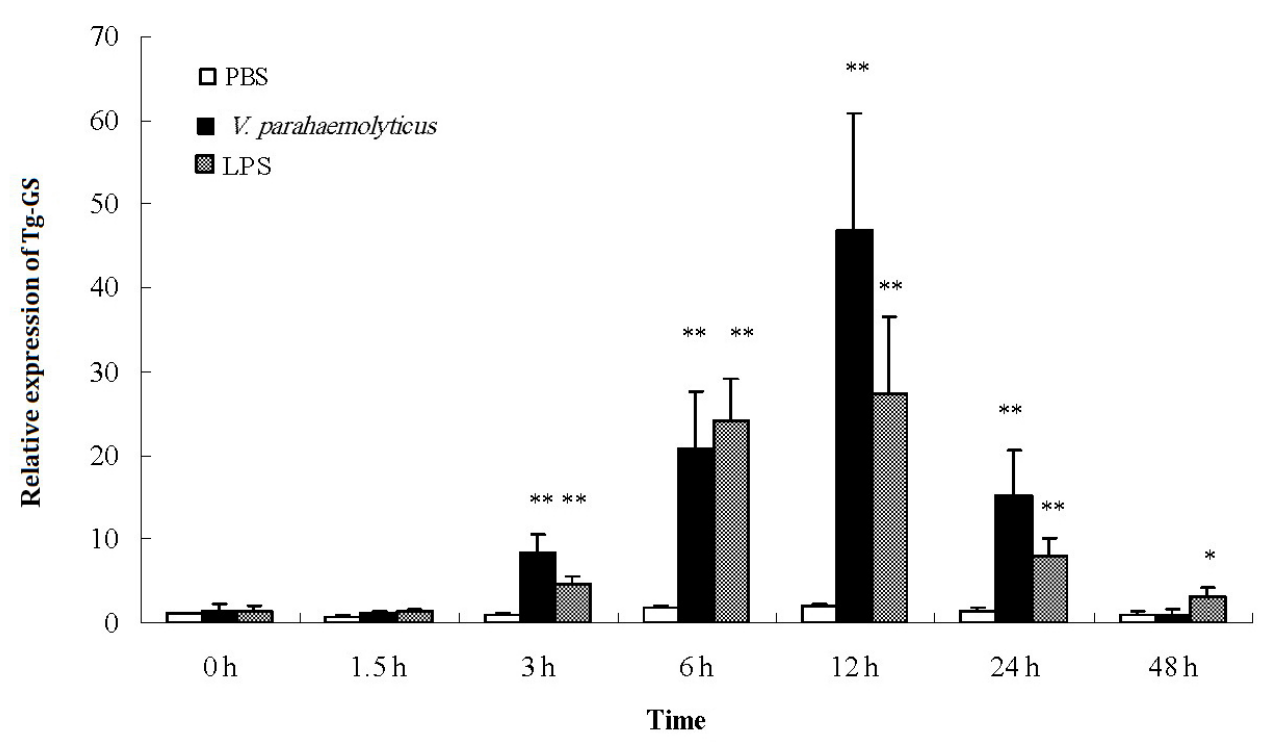

Figure 5. Temporal expression of glutamine synthetase of Tegillarca granosa (Tg-GS) in haemocytes of blood clam injected with Vibrio parahaemolyticus and lipopolysaccharide (LPS). For each time point, Tg-GS transcripts were quantified using real-time RT-PCR from 4 individuals. The amount relative to the internal control 18srRNA gene is expressed as means \pm standard deviation $(\mathrm{N}=4)$. Comparison among different time points was performed using the Fisher LSD $t$-test. Results that are significantly different $(\mathrm{P}<0.05)$ from time 0 are indicated with an asterisk; results that are very significantly different $(\mathrm{P}<0.01)$ are indicated with two asterisks.

In the $V$. parahaemolyticus challenge group, Tg-GS transcripts increased gradually from 3 to $12 \mathrm{~h}$ post-injection, reaching the highest level $12 \mathrm{~h}$ post-injection $(\mathrm{P}<0.01)$, decreasing gradually from 12 to $48 \mathrm{~h}$, and returning to original levels at $48 \mathrm{~h}$. The results in the LPS stimulation group were similar to those of the $V$. parahaemolyticus group, but the highest expression at $12 \mathrm{~h}$ was lower than that at the same time in the V.parahaemolyticus group, and it recovered to twice the original level at $48 \mathrm{~h}$. No significant change $(\mathrm{P}>0.05)$ in the control group occurred throughout the experiment. The mortality of the infected group was $4 \%$ and that of the control group (injected with PBS) was $0 \%$.

\section{DISCUSSION}

We determined the full-length cDNA of Tg-GS. The multiple alignment of the deduced amino acid sequence of Tg-GS with that of other organisms revealed that principal domains, $\mathrm{Mg}^{2+}$ ion binding sites, and ATP binding sites were completely conserved among the selected vertebrates and invertebrates. This conserved sequence and motif suggested that TgGS may have the same function in all organisms. These conserved amino acids were likely to stabilize GS structure and function in evolutionary terms. ATP and $\mathrm{Mg}^{2+}$ must bind to GS to activate it; then, it can bind to glutamic acid (Hayward et al., 1986; Tanguy et al., 2005). Tg-GS has several ATTTA $(G)$ motifs in a 3'-UTR, as do other species. The 3'-UTR length of $572 \mathrm{bp}$ is longer than those in the Pacific oyster (280 bp) and sea anemone (Aiptasia pallida; $450 \mathrm{bp}$ ) (Smith et al., 2004) but shorter than those of other invertebrates such as D. melanogaster and 
A. aegypti (Caizzi et al., 1990; Smartt et al., 2001). Only one AATAAA was found in Tg-GS, suggesting that this transcript encodes only one protein. Mammals, fish, and birds also display only a single GS gene (Kuo and Darnell Jr., 1989; Wang et al., 1996), but some invertebrates, such as D. melanogaster, display two GS isozymes (Caizzi et al., 1990).

The topology of the phylogenetic tree distinguished two GS subgroups - vertebrate GSs and invertebrate GSs - in general agreement with the taxonomic classification of the corresponding species. This high divergence between animal GSs and plant GSs might indicate the early differentiation of GS protein evolution and potential differences in biological functions. However, the lack of GS sequences from molluscs and invertebrates, in general, prevents a more complete evolutionary analysis.

Real-time PCR analysis demonstrated that Tg-GS mRNA was expressed in all studied tissues. The universal distribution of Tg-GS and its high expression level in hemocytes and mantle were particularly intriguing given their potentially important roles in the innate immune system of the blood clam. In rainbow trout, the highest level of GS expression occurs in the brain, with decreasing levels in intestine, liver, red muscle, gill/kidney, white muscle, and heart (Murray et al., 2003). GS expression is high in neural tissues and in post-blood-fed female $A$. aegypti mosquito midguts, in which it is involved in peritrophic matrix formation (Avisar et al., 1999; Smartt et al., 2001). In plants, the evidence reported herein indicates that the pine GS gene family contains at least two isoforms that have unique and precise patterns of spatial and temporal expression, suggesting that they play distinct functional roles in nitrogen metabolism of conifers (Avila et al., 2001). However, no such detailed studies have occurred in animals, especially invertebrates. The data presented here suggest that GS genes are differentially expressed in various tissues and therefore possibly involved in different metabolic pathways. The higher transcriptional expression in hemocytes and mantle suggests that Tg-GS may be involved in immune reactions.

Diseases caused by pathogenic or opportunistic bacteria such as Vibrio spp are still considered a major hurdle in the sustainable development of aquaculture worldwide (Baruah et al., 2010). Expression profiles of Tg-GS in hemocytes after $V$. parahaemolyticus and LPS challenge are shown in Figure 5. In the $V$. parahaemolyticus challenge group, Tg-GS transcripts increased gradually from 3 to $12 \mathrm{~h}$ post-injection, peaking at $12 \mathrm{~h}$ post-injection ( $\mathrm{P}$ $<0.01$ ) and then decreasing over the next few hours. At $48 \mathrm{~h}$, the expression recovered to original levels. The LPS stimulation group showed results similar to those in the $V$. parahaemolyticus group, but the expression level was lower than that in the $V$. parahaemolyticus group although still obviously higher than that of the blank group at $48 \mathrm{~h}$. The results suggested that Tg-GS can have significant immune response in Vibrio challenge assays.

GS mRNA expression can be regulated by various physiological conditions or extra stimulation. In vertebrates, hormones such as insulin and hydrocortisone can induce changes in the rate of GS biosynthesis (Eisenberg et al., 2000). In the Pacific oyster, GS expression is highly regulated in xenobiotic-exposed oysters compared to that in controls for all treatments (Tanguy et al., 2005). In the sea urchin (Paracentrotus lividus), an increase in GS expression was observed from the two-blastomere stage to the blastula stage, followed by a decrease in GS expression until the prism stage embryo (Fucci et al., 1995). Our results indicated that Tg-GS is potentially involved in immune responses against Vibrio challenge. LPS, which constitutes the outer leaflet of the outer membrane of most Gram-negative bacteria, is referred to as an endotoxin (Wang and Quinn, 2010). LPS has virulence in bacteria and induces a strong 
response from normal animal immune systems. Therefore, a reasonable conclusion from our findings is that Tg-GS is upregulated immediately after LPS infection or Gram-negative bacterial infection. Our study showed that Tg-GS can be immediately induced by LPS and could be involved in immune response against Gram-negative bacteria.

In conclusion, a full-length cDNA of Tg-GS was cloned. Tg-GS expression in hemocytes infected with $V$. parahaemolyticus and LPS as well as the expression levels in various tissues from the clam were studied. The results indicated that Tg-GS is a constitutive and inducible protein and thus could play an important role in immune responses against Gramnegative bacterial infection. Further studies in our laboratory are aimed at elucidating the mechanisms of transcriptional control of Tg-GS and the possible association of SNPs with resistance/susceptibility to bacterial infection.

\section{ACKNOWLEDGMENTS}

Research supported by the National High-Tech R\&D Program of China (\#2012AA10A410), the National Science Foundation of China (\#31001097), the Chinese Agriculture Research System (\#CARS-48), the Ningbo Science and Technology International Cooperation Research Projects (\#2010D10017), the Zhejiang Provincial Top Key Discipline of Modern Microbiology and Application (\#KF2012002), and the Zhejiang Marine Biotechnology Innovation Team (\#ZMBIT, \#2012R10029).

\section{REFERENCES}

Avila C, Suarez MF, Gomez-Maldonado J and Canovas FM (2001). Spatial and temporal expression of two cytosolic glutamine synthetase genes in Scots pine: functional implications on nitrogen metabolism during early stages of conifer development. Plant J. 25: 93-102.

Avisar N, Shiftan L, Ben-Dror I, Havazelet N, et al. (1999). A silencer element in the regulatory region of glutamine synthetase controls cell type-specific repression of gene induction by glucocorticoids. J. Biol. Chem. 274: 1139911407.

Bao Y, Li L, Wu Q and Zhang G (2009a). Cloning, characterization, and expression analysis of extracellular copper/zinc superoxide dismutase gene from bay scallop Argopecten irradians. Fish Shellfish Immunol. 27: 17-25.

Bao Y, Li L, Xu F and Zhang G (2009b). Intracellular copper/zinc superoxide dismutase from bay scallop Argopecten irradians: its gene structure, mRNA expression and recombinant protein. Fish Shellfish Immunol. 27: 210-220.

Baruah K, Ranjan J, Sorgeloos P and Bossier P (2010). Efficacy of heterologous and homologous heat shock protein 70s as protective agents to Artemia franciscana challenged with Vibrio campbellii. Fish Shellfish Immunol. 29: 733-739.

Brown JR, Masuchi Y, Robb FT and Doolittle WF (1994). Evolutionary relationships of bacterial and archaeal glutamine synthetase genes. J. Mol. Evol. 38: 566-576.

Caizzi R, Bozzetti MP, Caggese C and Ritossa F (1990). Homologous nuclear genes encode cytoplasmic and mitochondrial glutamine synthetase in Drosophila melanogaster. J. Mol. Biol. 212: 17-26.

Eisenberg D, Almassy RJ, Janson CA, Chapman MS, et al. (1987). Some evolutionary relationships of the primary biological catalysts glutamine synthetase and RuBisCO. Cold Spring Harb. Symp. Quant. Biol. 52: 483-490.

Eisenberg D, Gill HS, Pfluegl GM and Rotstein SH (2000). Structure-function relationships of glutamine synthetases. Biochim. Biophys. Acta 1477: 122-145.

Fahrner J, Labruyere WT, Gaunitz C, Moorman AF, et al. (1993). Identification and functional characterization of regulatory elements of the glutamine synthetase gene from rat liver. Eur. J. Biochem. 213: 1067-1073.

Fucci L, Piscopo A, Aniello F, Branno M, et al. (1995). Cloning and characterization of a developmentally regulated sea urchin cDNA encoding glutamine synthetase. Gene 152: 205-208.

Hayward BE, Hussain A, Wilson RH, Lyons A, et al. (1986). The cloning and nucleotide sequence of cDNA for an amplified glutamine synthetase gene from the Chinese hamster. Nucleic Acids Res. 14: 999-1008.

Kumada Y, Benson DR, Hillemann D, Hosted TJ, et al. (1993). Evolution of the glutamine synthetase gene, one of the 
oldest existing and functioning genes. Proc. Natl. Acad. Sci. U. S. A. 90: 3009-3013.

Kuo CF and Darnell JE Jr (1989). Mouse glutamine synthetase is encoded by a single gene that can be expressed in a localized fashion. J. Mol. Biol. 208: 45-56.

Murray BW, Busby ER, Mommsen TP and Wright PA (2003). Evolution of glutamine synthetase in vertebrates: multiple glutamine synthetase genes expressed in rainbow trout (Oncorhynchus mykiss). J. Exp. Biol. 206: 1511-1521.

Myohara M, Niva CC and Lee JM (2006). Molecular approach to annelid regeneration: cDNA subtraction cloning reveals various novel genes that are upregulated during the large-scale regeneration of the oligochaete, Enchytraeus japonensis. Dev. Dyn. 235: 2051-2070.

Shatters RG and Kahn ML (1989). Glutamine synthetase II in Rhizobium: reexamination of the proposed horizontal transfer of DNA from eukaryotes to prokaryotes. J. Mol. Evol. 29: 422-428.

Smartt CT, Kiley LM, Hillyer JF, Dasgupta R, et al. (2001). Aedes aegypti glutamine synthetase: expression and gene structure. Gene 274: 35-45.

Smith OP, Marinov AD, Chan KM and Ferrier MD (2004). Cloning and sequencing of cDNA encoding glutamine synthetase from the sea anemone Aiptasia pallida. Hydrobiologia 530-531: 267-272.

Tanguy A, Boutet I and Moraga D (2005). Molecular characterization of the glutamine synthetase gene in the Pacific oyster Crassostrea gigas: expression study in response to xenobiotic exposure and developmental stage. Biochim. Biophys. Acta 1681: 116-125.

Trapido-Rosenthal HG, Linser PJ, Greenberg RM, Gleeson RA, et al. (1993). cDNA clones from the olfactory organ of the spiny lobster encode a protein related to eukaryotic glutamine synthetase. Gene 129: 275-278.

Wang X and Quinn PJ (2010). Lipopolysaccharide: Biosynthetic pathway and structure modification. Prog. Lipid Res. 49: 97-107.

Wang Y, Kudoh J, Kubota R, Asakawa S, et al. (1996). Chromosomal mapping of a family of human glutamine synthetase genes: functional gene (GLUL) on 1q25, pseudogene (GLULP) on 9p13, and three related genes (GLULL1, GLULL2, GLULL3) on 5q33, 11p15, and 11q24. Genomics 37: 195-199. 